\title{
COMPARISON OF EFFECTIVENESS BETWEEN DPP4 INHIBITORS COMBINED WITH VERSUS OTHER ORAL HYPOGLYCAEMIC AGENT(S) IN DIABETIC PATIENTS
}

\author{
COLONEL MD. SHAMEEM HAIDAR ${ }^{1}$
}

\begin{abstract}
:
Background: Diabetes is global health burden of disease that requires life-long pharmacological and non-pharmacological management to prevent complications such as cardiovascular disease, retinopathy, nephropathy, and neuropathy. Treatment of type 2 diabetes is based on an interplay of patient characteristics, severity of hyperglycemia and available therapeutic options. Metformin, sulfonylureas (SU) and DPP IV inhibitor are the most studied of the oral medications used worldwide. They play a prominent initial role in the type 2 diabetes treatment algorithm recommended by the several guideline. The growing evidence on new technologies and therapeutic interventions is rapidly expanding our knowledge and ability to manage diabetes and its complications; at the same time, however, it is challenge for physicians to select appropriate medication in appropriate dose for optimal patients care.
\end{abstract}

Objectives: To compare the safety and efficacy of the dipeptidylpeptidase-4 (DPP-4) inhibitors combination with other oral hypoglycaemic agent(s) in patients with type 2 diabetes and inadequate glycemic control.

Materials \& method: Study was conducted among 600 patients over a period of 24 months. All the patients were adult male and female type 2 diabetic patients who received regular oral anti-diabetic drug(s) and duration of T2DM for one year or more were enrolled for study. Total 150 cases were selected. Patients with Type 1 DM, pregnant women with DM and who was receiving injectable antidiabetic medications were excluded from this study. Detail demographic data were collected from the informant and recorded in structured case report form. Clinical examination and relevant investigations were done. Main outcome variable was Glycemic status (HbA1C, FBG, 2HABF). Effectiveness of drugs was evaluated by glycaemic status of the patients.

Result: Maximum number of patients (38.5\%) was between 31-40 years age group with mean age $37.8 \pm 9.5$ years. Present study shows that, for good glycemic control, all three results (FBS, $2 \mathrm{H} \mathrm{ABF}$ and $H b A 1 c)$ were within targeted level in majority patients of DPP4 Inhibitor combination group. Although FBS was best result in metformin group. About $51.9 \%$ of SUs group achieved the glycemic control targets level. In case of metformin group it was in $59.8 \%$ of patients, and in combined therapy $67.1 \%$ patients shows good glycemic target. So DPP4 Inhibitor combination is better medication than other to maintain good glycemic status in type 2 DM patient, due to maximum number of patients reached all three components of result within target range.

Conclusion: Diabetes is chronic illness. Good glycemic control with choosing appropriate anti-diabetic medication is pivotal for DM management. In this study it is observed that DPP4 Inhibitor combination group of drug is better than other anti-diabetic medication to maintain good glycemic status in type 2 DM patients.

Keywords: Diabetes mellitus, Oral anti-diabetic medication, Sulphonylureas, Biguanide, Incretinmimetics (DPP IV inhibitor).

Received: 05 November 2018

Accepted: 11 April 2019

DOI: https://doi.org/10.3329/bjmed.v30i2.41532

Address of Correspondence: Dr.Colonel Md. Shameem Haidar, Classified Specialist in Medicine, CMH Dhaka Cantonment.

Bangladesh J Medicine 2019; 30 : 63-70 


\section{Introduction:}

Type 2 diabetes is one of the major public health concerns in both developing and developed countries. It has become epidemic in a number of countries, particularly in newly industrialised nations. The direct and indirect social and economic costs of treating diabetes and its complications have the potential to cripple the countries' healthcare budgets. In recent times, a new dimension has been added with the increasing appearance of type 2 diabetes in adolescents and even children. Good glycemic control can delay the progression of microvascular or macrovascular complication with in type 2 diabetes mellitus (T2DM) patients. To date, the association between antidiabetic agents and glycemic control in this patient is not well established.

Like many other developing countries, Bangladesh has a major problem of rapid urbanization. Dietary habit, life style modification also changed with urbanization. Any country that experienced to urbanization and industrialization also witnessed with a change of disease pattern from infectious to non-communicable and chronic diseases. Changing in life styles, eating habits, sedentary life, increased use of tobacco and deteriorating environmental conditions are likely to develop non-communicable diseases. At the same time non-communicable diseases and metabolic disorders linked with diabetes (DM), hypertension (HTN) and coronary heart diseases (CHD) are increasing. At present it is estimated that about 7.1 million cases of diabetes in Bangladesh in 2015 and latest data suggested that prevalence of diabetes in adult (20-79 years) is $7.4 \% .^{1}$

Type 2 Diabetes is defined as chronic hyperglycemia resulting from either decreased insulin secretion, impaired insulin action or both in the absence of autoimmune destruction of the pancreatic beta cell. Type 2 diabetes is now recognized as a major chronic public health problem throughout the world. It affects large number of people of wide range of ethnic and economic levels in both developed and developing countries. ${ }^{2}$ Diabetes mellitus can lead to diabetes specific complications and end organ damage which can be prevented or delayed by strict glycemic control and thereby reduce morbidity and mortality among these patients. In a major proportion of patients, oral anti-diabetic drugs are used for glycemic control early in disease course and are effective in many cases to reach the therapeutic targets. Summaries of previous studies of oral antidiabetic drugs (OADs) suggest that they reduce A1C levels by $0.5-1.25 \% .^{3}$ These oral medications are easy to take and often cost effective in comparison with injectable medications.
Essential components of the treatment for diabetes include diabetes self-management education and support, lifestyle interventions, and goal setting and glycemic management. Each class of oral agents works by a different mechanism and they may be combined to achieve optimal glucose control. The obvious exceptions are sulfonylureas and non-sulfonylurea insulin secretagogues, which should not be combined. ${ }^{4}$ Typically, patients with type 2 diabetes are started on metformin, with a second agent or third agent added as needed. In general, the addition of an oral agent will reduce $\mathrm{HbA} 1 \mathrm{c}$ by an additional $1.0 \%$. Tablets combining two classes of oral agents are now available.

People with type 2 diabetes often have lifestyles (eating and physical activity habits) which contribute to their problem. Begin oral glucose lowering medications when lifestyle interventions alone are unable to maintain blood glucose control at target levels. First-line therapy begin with metformin unless there is evidence of renal impairment or other contraindication. ${ }^{5}$ Other options include a sulfonylurea (or glinide) for rapid response where glucose levels are high, or a-gIucosidase inhibitors in some populations; these agents can also be used initially where metformin cannot. In some circumstances dual therapy may be indicated initially if it is considered unlikely that single agent therapy will achieve glucose targets.

There are several groups of oral medication available, currently five classes of OAD agents commonly used: Alpha glucosidase inhibitors (AGIs), Biguanides, Dipeptidyl peptidase-4 (DPP-4) Inhibitors, Insulin Secretagogues - SUs, Non-SUs or Meglitinides and Thiazolidinediones (TZDs). AGIs primarily lower postprandial glucose without causing hypoglycaemia. They are less effective in lowering glycaemia than metformin or $\mathrm{SU}$, reducing $\mathrm{HbA} 1 \mathrm{c}$ by $0.5-0.8 \%$. Metformin does not stimulate insulin secretion, and lowers blood glucose by decreasing hepatic glucose production. Metformin monotherapy is usually not accompanied by hypoglycaemia. It can lower plasma glucose by up to $20 \%$ as first line drug treatment especially in overweight/obese patients. Metformin monotherapy will lower HbA1c by about $1.5 \% .^{6}$ Dipeptidyl peptidase-4 (DPP-4) Inhibitor (Sitagliptin) lowers $\mathrm{HbA} 1 \mathrm{c}$ by $0.5-0.8 \%$, its efficacy improves when used at higher HbA1c baselines. Thiazolidinediones are peroxisome proliferator-activated receptor-gamma agonists and act primarily by increasing insulin sensitivity of muscle, adipose tissue and liver to endogenous and exogenous insulin (insulin sensitizers).

Reaching Glycaemic Targets achieved by Self blood glucose monitoring (SBGM) routinely. Monitoring 
provides information on the effects of therapy, diet and physical activity. Those on OAD agents or diet need to check fasting and 2-hour PPG levels. HbA1c should be measured approximately every 3 to 6 months to ensure that glycaemic targets are being met. This reflects overall glucose control over a 3 month period with recommended target level of $6.5 \%$. To achieve a HbA1c $<6.5 \%$, aim for FPG or pre-prandial plasma glucose targets of 4.4 to $6.1 \mathrm{mmol} / \mathrm{L}$ and 2-hour PPG targets of 4.4 to $8.0 \mathrm{mmol} / \mathrm{L}^{6}$

$\mathrm{HbA} 1 \mathrm{c}$ is the most commonly accepted measurement of long-term glycemic control. Current recommendations are that $\mathrm{HbA} 1 \mathrm{c}$ be checked at least every 6 months ${ }^{4}$ if the patient is well controlled (HbA1c d" $7 \%$ ) and on a stable oral anti-hypoglycemic regimen, otherwise every 3 months. Targets for therapy have been evaluated in clinical trials. Two trials have achieved A1c levels slightly greater than 7\%. Neither showed reduction in end-stage complications in the time frame of the trials (e.g., visual loss, renal failure amputation). However, early and intermediate microvascular complications were reduced, and longerterm follow-up of one study showed that benefits did begin to accrue by $15-20$ years. This suggests that a target $\mathrm{A} 1 \mathrm{c}$ of $7 \%$ to $7.5 \%$ is reasonable in those with life expectancies in this range or longer. ${ }^{4}$

Glycosylated hemoglobin assays provide an accurate indication of long-term glycemic control. Glycated hemoglobin is formed by the continuous non-enzymatic glycosylation of hemoglobin throughout the lifespan of an erythrocyte. The A1c assay yields an accurate measure of time-averaged blood glucose during the previous six to eight weeks. Clinically, it can assist in determining duration and severity of hyperglycemia and can help guide treatment. Eating, physical activity or acute metabolic stress does not influence the A1c test. The test can be done at any time of day and does not require fasting. ${ }^{7}$

Appropriate medication management targeting glycemic control, hypertension, and lipid management is important for reducing morbidity and mortality, and improving long-term quality of life for patients diagnosed with type 2 diabetes mellitus (T2DM). Lifestyle changes such as nutrition therapy, weight loss, increased exercise, and appropriate education and self-management strategies are pivotal to improved outcomes. Inadequate access to care for chronic disease management as well as the cost of medication can contribute to poor control of T2DM and associated cardiovascular risk factors. ${ }^{7}$

\section{Methodology:}

This quasi experimental Phase IV clinical trial was conducted amongst 600 patients of type 2 DM over a period of 24 months. Samples were selected by quasi experimental type. Sociodemographic variables were age, sex, economic status, educational level, etc. Clinical variables were glycemic status: $\mathrm{HbA} 1 \mathrm{C}, \mathrm{FBG}$, $2 \mathrm{HABF}$, Oral anti-diabetic agents. Informed written consent was obtained accordingly. The trial was done according to ICH-GCP and followed the declaration of Helsinki.The patients allocation was semi purposive and not randomized. The drugs which were prescribed was sulfonylureas (S), Biguanides (B) (Metformin) and DPP4 inhibitors(D) (Vildagliptin and Sitagliptin). There was no concealment of allocation and sequential allocation by $\mathrm{S}, \mathrm{B}$ and $\mathrm{D}$ groups. The combination was done with Vildagliptin and metformain $(V+M)$ and sitagliptin and metformin $(\mathrm{S}+\mathrm{M})$. So the allocation was 4 cards with one $\mathrm{S}$, One B, One with $\mathrm{S}+\mathrm{M}$ and one with $\mathrm{V}+\mathrm{M}$ Primary efficacy was seen of glycaemic control and secondary efficacy was to see the achievement of target glucose. The safety issue was seen with primarily see the number of hypoglycaemic episodes and secondary to see the number of death. All tests were done in recognized Biochemistry lab. Reference range of investigation report obtained according to operational definition. This CRF was used for collection of information by interviewing patients. Data processing work consist of registration schedules, editing computerization, preparation of dummy table, analyzing and matching of data. Quantitative data expressed as mean and standard deviation and qualitative data as frequency and percentage. Comparison was done by tabulation and graphical presentation in the form of tables, pie chart, graphs, bar diagrams, histogram \& charts etc.

\section{Operational definition:}

Diabetes mellitus (DM): The current WHO diagnostic criteria for diabetes

- $\quad$ Fasting blood glucose $\geq 7.0 \mathrm{mmol} / 1$

- $\quad$ Or 2-hours after ingestion of 75 gm glucose $\geq 11.1$ $\mathrm{mmol} / 1$

- $\quad$ Or $\mathrm{HbA} 1 \mathrm{C} \geq 6.5 \%$

In the presence of classical symptoms of hyperglycemia

Or, diagnosed case of T2DM on anti-diabetic medications

Glycemic control: Targets are-

- Fasting blood glucose: Â $6.0 \mathrm{mmol} / \mathrm{L}$

- Or HbA1c: Â 7.0\%

- Or 2-hour postprandial blood glucose: Â 8.0 $\mathrm{mmol} / \mathrm{L}$ 


\section{Result}

In this study Maximum number of patients (38.5\%) was between $31-40$ years age group with mean age $37.8 \pm 9.5$ years. Out of 600 cases, $57.6 \%$ cases were male and $42.3 \%$ were female. Male to female ratio was 1.3:1. (Table I) Large numbers of respondents came from urban area and poor class 66(44\%) comprising the major percentage of the patients. Majority of Diabetic patients were suffering for disease 1 to six year $(61 \%)$.

Patient's allocation according to prescribed medication revealed that, $198(43 \%)$ patients having history of taking sulphonylureas (SUs), 192(32.0\%) patients biguanide and $210(35.0 \%)$ patients having history of taking combination of DPP IV inhibitor. Combination therapy distributed as $118(19.6 \%)$ Vildagliptin \& metformin combination and 92(15.3\%) Sitagliptin \& metformin combination. (Table II).

Table I

Demographic profile of the patients $(n=600)$

\begin{tabular}{lccc}
\hline Age (yr.) & \multicolumn{2}{c}{ Frequency } & Total (\%) \\
\cline { 2 - 3 } & Male $(n=346)$ & female $(n=254)$ \\
\hline d"30 & $98(28.3)$ & $51(20.0)$ & $149(24.8)$ \\
$31-40$ & $139(40.1)$ & $92(36.2)$ & $231(38.5)$ \\
$41-50$ & $71(20.5)$ & $60(23.6)$ & $131(21.7)$ \\
$>50$ & $38(10.9)$ & $51(20.0)$ & $89(14.6)$ \\
Mean \pm SD & $38.1 \pm 9.2$ & $37.5 \pm 11.4$ & $600(100)$ \\
\hline M:F & \multicolumn{3}{c}{$1.3: 1$} \\
\hline
\end{tabular}

Table II

Patients allocation according to types of oral OAD medication $(n=600)$

\begin{tabular}{llcc}
\hline OAD agent(s) & Frequency & Percentage \\
\hline SUs & Glimepiride & 54 & 9.0 \\
& Gliclazide & 64 & 10.6 \\
& Glipizide & 20 & 3.3 \\
& Glybenclamide & 60 & 10.0 \\
Biguanide & Metformin & 192 & 32.0 \\
DPP4 & Vildagliptin \& & 118 & 19.6 \\
Inhibitor & metformin & & \\
& Sitagliptin \& & 92 & 15.3 \\
& metformin & & \\
\hline
\end{tabular}

Regarding the operational definition of glycemic control targets, target level of fasting blood glucose Â6.0 mmol/ $\mathrm{L}$ is considered as good status. Present study revealed that metformin (Biguanide group) observed good glycemic control in $129(67.1 \%)$ patients, followed by Vildagliptin \& metformin combination in $72(61.0 \%)$ patients, Sitagliptin \& metformin combination in 54(58.6\%) patients, Gliclazide 37(57.8\%) patients and Glimepiride 31(57.4\%) patients. Overall in case of SUs group result was $108(54.5 \%)$ of patients and in combination DPP4 Inhibitor group was $126(60.0 \%)$ of patients of good glycemic status. (Table III)

\section{Table III}

Evaluation of glycemic status by FBG level in different groups $(n=600)$

\begin{tabular}{lcc}
\hline OAD agent(s) & \multicolumn{2}{c}{ FBG (mmol/L) } \\
\cline { 2 - 3 } & $<6.0$ & $\geq 6.0$ \\
\hline Glimepiride & $31(57.4 \%)$ & $23(42.5 \%)$ \\
Gliclazide & $37(57.8 \%)$ & $27(42.1 \%)$ \\
Glipizide & $8(40 \%)$ & $12(60 \%)$ \\
Glybenclamide & $32(53.33 \%)$ & $28(46.66 \%)$ \\
Metformin & $129(67.1 \%)$ & $63(32.8 \%)$ \\
Vildagliptin \& metformin & $72(61.0 \%)$ & $46(38.9 \%)$ \\
Sitagliptin \& metformin & $54(58.6 \%)$ & $38(41.3 \%)$ \\
\hline
\end{tabular}

According to the targeted FBG, Bar chart shows that patients of metformin group achieved better glycemic status then others in T2DM patient. The $p$-value is 0.000625 . The result is significant at $p<.05$. (Figure-1).

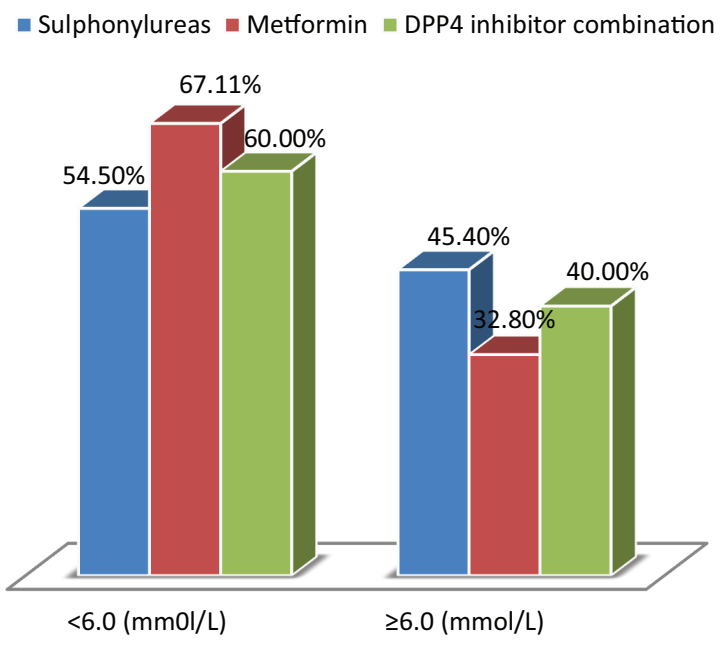

Fig.-1: Assessment of effectiveness of drugs amongst the groups by the result of FBG $(n=600)$

2-hour postprandial blood glucose less than $8.0 \mathrm{mmol} /$ $\mathrm{L}$ was indicator for good glycemic status. In this study, combination therapy by Vildagliptin \& metformin 
combination was observed good glycemic control than others, reported in $84(71.1 \%)$ patients, followed by Glimepiride in $38(70.3 \%)$ patients, Sitagliptin \& metformin combination in 59(64.1\%) patients and metformin (Biguanide group) in 117(60.9\%). Overall findings suggested that in case of SUs group result was $109(55.0 \%)$ of patients, metformin group was $117(60.9 \%)$ and in combination DPP4 Inhibitor group was $143(68.0 \%)$ of patients of post-prandial good glycemic status. (Table IV)

Table IV

2-hours after breakfast blood glucose levels in different groups $(n=600)$

\begin{tabular}{lcc}
\hline OAD agent(s) & \multicolumn{2}{c}{$2 \mathrm{H} \mathrm{ABF} \mathrm{(mmol/L)}$} \\
\cline { 2 - 3 } & $<8.0$ & $\mathrm{e}-8.0$ \\
\hline Glimepiride & $38(70.3 \%)$ & $16(29.6 \%)$ \\
Gliclazide & $33(51.5 \%)$ & $31(48.4 \%)$ \\
Glipizide & $9(45.0 \%)$ & $11(55.0 \%)$ \\
Glybenclamide & $29(48.3 \%)$ & $31(51.6 \%)$ \\
Metformin & $117(60.9 \%)$ & $75(39.0 \%)$ \\
Vildagliptin \& metformin & $84(71.1 \%)$ & $34(28.8 \%)$ \\
Sitagliptin \& metformin & $59(64.1 \%)$ & $33(35.8 \%)$ \\
\hline
\end{tabular}

Present study shows that effectiveness of SUs group and metformin group almost similar in the means of result of post-prandial blood glucose level, (55.0\%) and $(60.9 \%)$ respectively. But result of combination therapy DPP4 was better than others. The $p$-value is 0.000371 . The result is significant at $p<.05$. (Figure-2)

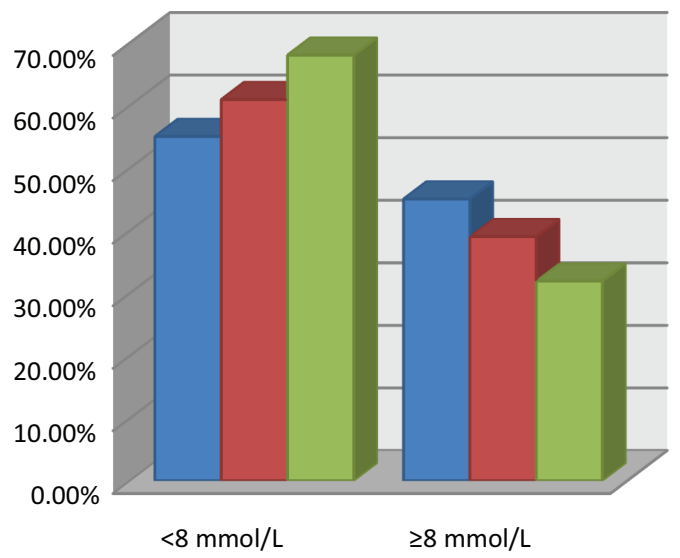

Sulphonylureas $\square$ Metformin $\square$ DPP4 inhibitor combination

Fig.-2: Assessment of effectiveness of drugs amongst the groups by the result of $2 \mathrm{H} \mathrm{ABF}(n=600)$
Table V

$H b A 1 c$ levels in the study population $(n=600)$

\begin{tabular}{lccc}
\hline OAD agent(s) & \multicolumn{3}{c}{ HbA1C } \\
\cline { 2 - 4 } & $<7.0 \%$ & $7.1 \%$ to $9.0 \%$ & $>9 \%$ \\
\hline Glimepiride & $32(59.2 \%)$ & $19(35.1 \%)$ & $3(5.5 \%)$ \\
Gliclazide & $33(51.5 \%)$ & $31(48.4 \%)$ & 0 \\
Glipizide & $8(40.0 \%)$ & $10(50.0 \%)$ & $2(10.0 \%)$ \\
Glybenclamide & $29(48.3 \%)$ & $29(48.3 \%)$ & $2(3.3)$ \\
Metformin & $115(59.8 \%)$ & $72(37.5 \%)$ & $5(2.6 \%)$ \\
Vildagliptin \& & $82(69.4 \%)$ & $36(30.5 \%)$ & 0 \\
metformin & & & $2(2.1 \%)$ \\
Sitagliptin \& & $59(64.1 \%)$ & $31(33.6 \%)$ & \\
metformin & & & \\
\hline
\end{tabular}

$\mathrm{HbA} 1 \mathrm{c}$ is the most commonly accepted measurement of long-term glycemic control. Regarding the operational definition acceptable level of HbA1c is Â7.0\% for strict glycemic control. Present study shows that, in SUs group 102(51.5\%) of patients had good glycemic control. In metformin group $115(59.8 \%)$ of patients and in DPP4 Inhibitor combination group $141(67.1 \%)$ of patients under control of strict level. So present study suggested that, DPP4 Inhibitor combination therapy is best medication for control of strict glycemic status. . (Table V)

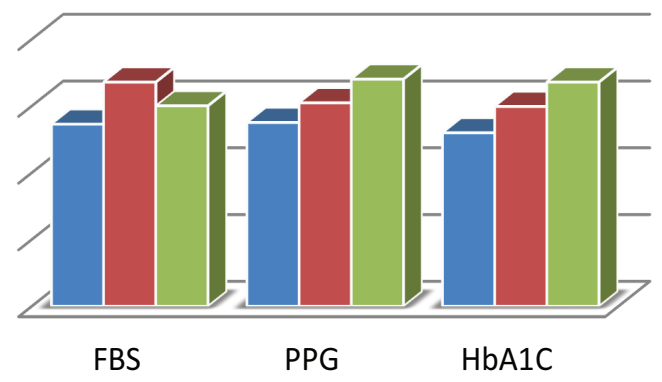

- Sulphonylureas - Metformin DPP4 Combined therapy

Fig.-3: Comparison of effectiveness of drugs amongst the groups by the result of FBS, $2 \mathrm{HABF}, \mathrm{HbA1c}(\mathrm{n}=600)$

All findings were evaluated in all groups of patients. Present study shows that, for good glycemic control, all three results (FBS, $2 \mathrm{H} \mathrm{ABF}$ and $\mathrm{HbA} 1 \mathrm{c}$ ) were within targeted level in majority patients of DPP4 Inhibitor combination group. Although FBS was best result in metformin. About $51.9 \%$ of SUs group achieved the glycemic control targets level. In case of metformin group it was in $59.8 \%$ of patients, and in combined therapy $67.1 \%$ patients shows good glycemic target. 
So DPP4 Inhibitor combination is better medication than other to maintain good glycemic status in type 2 DM patient, due to maximum number of patients reached all three components of result within target range. The $p$-value is 0.023685 . The result is significant at $p<.05$. (Figure-3).

\section{Discussion:}

In this quasi experimental Phase IV Clinical trial we investigated 600 individuals type 2 DM both male and female $>20$ years of age. For estimating the glycemic status of diabetes we evaluated fasting blood glucose (FBG), 2-hr post glucose test and HbA1c for selected subjects. We also measured some important sociodemographic and socio-economic information following a pre-tested structured case record form. For diagnosis and defining the diabetes state in our study population we used revised WHO criteria. In this study Maximum number of patients $(38.5 \%)$ was between $31-40$ years age group with mean age $37.8 \pm 9.5$ years. Male to female ratio was 1.3:1.

Findings are correlates with the results of similar studies at home and abroad, e.g a cross- sectional study in outpatients medicine department ${ }^{8}$ reported that the mean ages (in years) of the male and female diabetic patients were $51.5 \pm 1.51$ and $50.4 \pm 1.46$. A study in Bangladesh reported that among study subjects 731 were male, 824 were female, most of the population were young with a mean age 33 years, and about 78 percent were in age category between $20-40$ years $^{2}$. Another study in Bangladesh by Akter S; et al. reported that diabetes and prediabetes are highly prevalent among individuals aged 35 years or more in Bangladesh ${ }^{9}$. Another study by Rahman H (2012) ${ }^{10}$ showed a significant difference $(\mathrm{M}: \mathrm{F}=2: 1)$ with higher male preponderance. It is found that the incidence of DM rose with the increase of age and declined sharply after the age of 50 years.

In this study, patient's allocation according to prescribed medication revealed that, 198(43\%) patients having history of taking sulphonylureas (SUs), $192(32.0 \%)$ patients biguanide and $210(35.0 \%)$ patients having history of taking combination of DPP IV inhibitor. Combination therapy distributed as $118(19.6 \%)$ Vildagliptin \& metformin combination and 92(15.3\%) Sitagliptin \& metformin combination.

In patients with type 2 diabetes, diet and physical activity are essential first line therapies, and many groups now recommend initiating metformin at diagnosis. Study reported Metformin should be prescribed as the first line agent unless there are contradictions to its use ${ }^{4}$. The choice of subsequent agents remains controversial. Sulfonylureas should be considered as a second-line agent. Weight-neutral medications have clinical appeal, but no outcomes data to support their use over any other medication. In general, if the patient has not achieved glycemic goal after therapy at a maximal dose of an oral agent, the therapy should be considered inadequate and combination therapy may be chosen. In this study total $210(35.0 \%)$ patients had prescribed combination of DPP IV inhibitor.

Regarding the operational definition of Glycemic control targets, target level of fasting blood glucose was: Â6.0 $\mathrm{mmol} / \mathrm{L}$. Present study revealed that patients treated with metformin (biguanide group) had good glycemic control $129(67.1 \%)$ in the means of level of fasting blood glucose (Â6.0mmol/L), followed by combination therapy of DPP IV inhibitor in 126(60.0\%) patients. According to the targeted FBG, bar chart shows that patients of metformin group achieved better glycemic status then others in T2DM patient. The $p$-value is 0.000625 . The result is significant at $p<.05$. A study reported that metformin has excellent efficacy with favorable weight and lipid profiles supporting its use as first-line therapy. DPP-4 inhibitors are less efficacious than metformin but have proven noninferior to SU treatment with a favorable side-effect profile and less risk for hypoglycemia 9 .

In this study, 2-hour postprandial blood glucose less than $8.0 \mathrm{mmol} / \mathrm{L}$ was indicator for good glycemic status. In this study, combination therapy by Vildagliptin \& metformin combination was observed good glycemic control than others, reported in $84(71.1 \%)$ patients, followed by Glimepiride in $38(70.3 \%)$ patients, Sitagliptin \& metformin combination in $59(64.1 \%)$ patients and metformin (Biguanide group) in $117(60.9 \%)$. Overall findings suggested that in case of SUs group result was $109(55.0 \%)$ of patients, metformin group was $117(60.9 \%)$ and in combination DPP4 Inhibitor group was $143(68.0 \%)$ of patients of post-prandial good glycemic status. So patients with combination therapy by Vildagliptin \& metformin shows best postprandial blood glucose achievement than others.

Present study shows that, for good glycemic control, all three results (FBS, $2 \mathrm{H} \mathrm{ABF}$ and $\mathrm{HbA} 1 \mathrm{c}$ ) were within targeted level in majority patients of DPP4 Inhibitor combination group. Although FBS was best result in metformin. About $51.9 \%$ of SUs group achieved the glycemic control targets level. In case of metformin group it was in $59.8 \%$ of patients, and in combined therapy $67.1 \%$ patients shows good glycemic target. So DPP4 Inhibitor combination is better medication than other to maintain good glycemic status in type 2 DM patient, due to maximum number of patients 
reached all three components of result within target range.

All findings consistent with result of other studies. In a systematic review of randomized controlled trials (RCTs) shows that all DPP-4 inhibitors plus metformin were significantly more effective than metformin alone in reducing mean $\mathrm{HbA} 1 \mathrm{c}$ from baseline and achieving a higher proportion of patients with $\mathrm{HbA} 1 \mathrm{c}<7 \%{ }^{11}$. Dipeptidylpeptidase-4 (DPP-4) inhibitors have a mechanism of action that is distinct from other oral glucose-lowering agents ${ }^{12}$. The DPP-4 inhibitor class of oral anti-diabetic agents selectively inhibits the DPP4 enzyme that rapidly degrades two major incretin hormones, glucagon-like peptide-1 (GLP-1) and glucose-dependent insulinotropic polypeptide ${ }^{13,15}$.

DPP-4 inhibitors have also been shown to be efficacious and safe in combination therapy with metformin, SUs, TZDs and insulin ${ }^{15}$. Scheen ${ }^{13}$ reviewed DPP-4 inhibitors in 2011, analyzing the similarities and differences among members of the DPP-4 inhibitor class of oral anti-diabetic agents, including their efficacy and safety profiles as monotherapy or in combination with metformin, a sulfonylurea (SU) and/ or a thiazolidinedione, and insulin. The review demonstrated that, although DDP-4 inhibitors produce a similar reduction in glycosylated hemoglobin (HbA1c) levels compared with other existing classes of oral glucose-lowering agents, DPP-4 inhibitors offer several clinical advantages ${ }^{14}$. These include negligible risk of hypoglycemia, much lower than that observed with SUs, and weight neutrality, compared with the weight gain that is generally associated with SUs and thiazolidinediones ${ }^{13}$.

The pharmacokinetics across the DPP-4 class of oral diabetes medications is, in general, similar with a few exceptions. The agents in this class have good oral bioavailability which remains essentially unchanged with food intake. The relatively long half-lives of sitagliptin, linagliptin and alogliptin allow for once-daily dosing. Saxagliptin and vildagliptin both have shorter half-lives but saxagliptin is still dosed once daily because of its active metabolite while vildagliptin is dosed twice daily (b.i.d.) ${ }^{12}$. Based on the above knowledge, the combination of DPP-4 inhibitor suggested as a promising treatment strategy for improving glycemic control while attenuating insulinrelated risks in T2D patients.

\section{Conclusion:}

A The principal finding of this study is DPP-4 inhibitors combination therapy is the best medication across the class in terms of key efficacy and safety outcomes $\left(\mathrm{HbA}_{1 \mathrm{c}}, \mathrm{FBS}, \mathrm{PPG}\right.$ and proportion of patients achieving
$\left.\mathrm{HbA}_{1 \mathrm{c}}<7 \%\right)$. Glycaemic control would prevent microvascular and macrovascular complications of type 2 diabetes. Several categories of oral antidiabetic drugs including biguanides, sulfonylureas, meglitinides, DPP-4 (dipeptidyl peptidase-4) inhibitors are available for tretment of type 2 diabetes. Efficacies of these drugs should be monitored for clinical evaluation and for further management. Other oral agents, like metformin are less efficacious than DPP-4 inhibitors combination but have proven non-inferior to SU treatment with a favorable side-effect profile and less risk for hypoglycemia. In conclusion, our data indicate that the combination treatment of DPP-4 inhibitor is beneficial in terms of glycemic control. However, additional studies are warranted to establish the optimal approach for its application in clinical practice.

\section{References}

1. Scorecard in Bangladesh. International Diabetes Federation. 2015. Available at http://www.idf.org/ membership/sea/bangladesh. Retrieved on March 2016.

2. Rahim MA. Diabetes in Bangladesh: Prevalence and Determinants. A thesis for the degree of Master of Philosophy in International Community Health. University of Oslo, Norway, June 2002

3. SherifaliD, Nerenberg K, Pullenayegum E et al. The Effect of Oral Anti-diabetic Agents on A1C Levels. A systematic review and meta-analysis. Diabetes Care 2010, 33:1859-1864 https://doi.org/10.2337/dc091727. PMid:20484130 PMCid:PMC2909079

4. Standiford CJ, Vijan S, Choe H et al; UMHS Management of Type 2Diabetes Mellitus, Guidelines for Clinical Care Ambulatory. May 2014. Page 1-29

5. Clinical Guidelines Task Force: Global Guidelinefor Type 2 Diabetes. International Diabetes Federation, 2012. Available at www.idf.org. Sec1:1 (5 / 123) to Sec2:117 (120 / 123). Retrieved on March 2016.

6. Clinical Practice Guidelines (CPG) on Management of Type 2 Diabetes Mellitus (T2DM). May 2009. Page 1-73

7. Redmon B, Caccamo D, Flavin P, Michels R, O'Connor $\mathrm{P}$, Roberts $\mathrm{J}$ et al. Institute for Clinical Systems Improvement. Diagnosis and Management of Type 2Diabetes Mellitus in Adults. Updated July 2014. Page $1-86$.

8. Shabana S. Uric Acid in T2DM Associated with Hypertension. Journal of Clinical and Diagnostic Research.2012; September (Suppl), Vol-6(7): 11401143

9. Akter S, Rahman M, Abe S, Sultana P. Prevalence of diabetes and pre-diabetes and their risk factors among Bangladeshi adults: a nationwide survey. Bull World Health Organ 2014;92:204-213A. doi: http:// dx.doi.org/10.2471/BLT.13.128371 https://doi.org/ 10.2471/BLT.13.128371 
10. Rahman H. Study of serum uric acid level as a risk factor for ischemic stroke. FCPS dissertation, 2011.

11. Craddy $\mathrm{P}$, Palin $\mathrm{H}$, Johnson K. Comparative Effectiveness of Dipeptidylpeptidase-4 Inhibitors in Type 2 Diabetes: A Systematic Review and Mixed Treatment Comparison. Diabetes Ther (2014) 5:1-41. https://doi.org/10.1007/s13300-014-0061-3 PMid:24664619 PMCid:PMC4065303

12. Krentz AJ, Patel MB, Bailey CJ. New drugs for type 2 diabetes mellitus: what is their place in therapy? Drugs. 2008; 68(15): 2131-62. https://doi.org/10.2165/ 00003495-200868150-00005 PMid:18840004
13. Scheen AJ. A review of gliptins in 2011. Expert Opin Pharmacother. 2012; 13(1):81-99. https://doi.org/ 10.1517/14656566.2012.642866.PMid:22149369

14. Phung OJ, Scholle JM, Talwar M, Coleman CI. Effect of noninsulin antidiabetic drugs added to metformin therapy on glycemic control, weight gain, and hypoglycemia in type 2 diabetes. JAMA. 2010; 303(14): 1410-8. https://doi.org/10.1001/jama.2010.405 PMid:20388897

15. Aleskow S, Lamos E, Davis S. A review of the efficacy and safety of oral antidiabetic drugs. Expert Opin Drug Saf. 2013 March; 12(2): 153-175. https://doi.org/ 10.1517/14740338.2013.752813.PMid:23241069 PMCid:PMC3977601 